The authors conclude that expectant management should be further investigated as a treatment option for patients with lowrisk, superficial bladder tumors, and might be particularly useful for those at high risk of adverse effects from surgery.

Original article Pruthi RS et al. (2008) Conservative management of low risk superficial bladder tumors. J Urol 179: $87-90$

\section{Rapid intraoperative tissue expansion can be used for scrotal reconstruction}

Surgical reconstruction for extensive scrotal tissue loss usually requires scrotal approximation or tissue transplantation. Rapid intraoperative tissue expansion (RITE) involves swift, single-stage stretching of skin from local tissue, which facilitates a tension-free closure without the need for tissue transplantation. Although RITE is presently used for other reconstructions (e.g. facial and extremity defects), its genitourinary applications have not been extensively explored. Kwon and colleagues report their experience of RITE for scrotal reconstruction.

Ten patients underwent scrotal reconstruction for necrotizing fasciitis at the authors' center between 1996 and 2006, of whom three underwent the RITE procedure; these patients had defects affecting $70-80 \%$ of the scrotal skin, which ruled out tension-free closure. The RITE technique involved subdermal insertion of $300 \mathrm{ml}$ tissue expanders in the residual scrotal tissue and medial anterior thigh flaps, which were then inflated for $4 \times 5$ min periods, separated by 2 min rest intervals. The expanded skin was used to perform a layered midline closure of the scrotum.

All three patients had satisfactory functional and cosmetic results after surgery, and were discharged from hospital the following day. No postoperative complications were observed during the 12-month follow-up period; their pain was managed with NSAIDs, and no patient required narcotic analgesia.

The authors conclude that, in select cases of extensive, partial, scrotal skin defects, closure with the RITE technique is cosmetically and functionally comparable to that achieved with complex tissue transfer and staged tissue expansion; the procedure might also be useful in other genitourinary reconstruction settings.

Original article Kwon EO et al. (2008) Scrotal

reconstruction using rapid intraoperative tissue expansion: a preliminary report. J Urol 179: 207-209

\section{Good intermediate-term oncologic outcomes reported for RF-LPN}

The widespread adoption of laparoscopic partial nephrectomy (LPN) for the treatment of small renal tumors has been hindered by its inherent technical difficulties. Radiofrequencyassisted LPN (RF-LPN), a novel technique that simplifies standard LPN, involves coagulation of the tumor with a radiofrequency probe followed by laparoscopic excision; no occlusion of the renal hylum is required (thus eliminating warm ischemia), and only fibrin glue is used to prevent urinary leaks. Having previously reported good short-term oncologic outcomes with this technique, Zeltser and colleagues now report the first intermediate-term results from a prospective database of patients who underwent RF-LPN for renal masses.

The authors identified 30 patients (mean age 56 years, range $21-75$ years) who underwent RF-LPN for small renal masses (mean size $1.9 \mathrm{~cm}$ ). Follow-up evaluations, consisting of abdominal CT or MRI and chest X-ray, were performed at 6 and 12 months postoperatively and annually thereafter.

Of the 32 masses identified, 29 had $\geq 6$ months' follow-up data (mean 31 months, range 6-60 months) and were included in oncologic outcome analysis. Mean intraoperative blood loss was $80 \mathrm{ml}$; no procedures were converted to radical nephrectomy or open surgery, and none required hylar clamping. No local tumor recurrence or progression to metastatic disease was observed at the latest follow-up, yielding a cancer-specific survival of $100 \%$. Postoperative complications included transiently elevated plasma creatinine levels $(n=2)$, hydrocalyx $(n=1)$ and mild port-side cellulitis $(n=1)$.

The authors conclude that RF-LPN shows good intermediate-term cancer control and low postoperative complication rates.

Original article Zeltser IS et al. (2008) Intermediate-term prospective results of radiofrequency-assisted laparoscopic partial nephrectomy: a non-ischaemic coagulative technique. BJU Int 101: 36-38 